\title{
Increasing the Accuracy of Binary Neutron Star Simulations with an improved Vacuum Treatment
}

\author{
Amit Poudel ${ }^{1}$, Wolfgang Tichy ${ }^{1}$, Bernd Brügmann ${ }^{2}$, and Tim Dietrich ${ }^{3}$ \\ 1 Department of Physics, Florida Atlantic University, Boca Raton, FL 33431 USA \\ 2 Theoretical Physics Institute, University of Jena, 07743 Jena, Germany and \\ 3 Institute of Physics and Astronomy, University of Potsdam, 14476 Potsdam, Germany
}

(Dated: September 15, 2020)

\begin{abstract}
Numerical relativity simulations are essential to study the last stages of the binary neutron star coalescence. Unfortunately, for stable simulations there is the need to add an artificial low-density atmosphere. Here we discuss a new framework in which we can effectively set the density surrounding the neutron stars to zero to ensure a more accurate simulation. We test our method with a number of single star test cases and for an equal mass binary neutron star simulation. While the bulk motion of the system is not influenced, and hence, there is no improvement with respect to the emitted gravitational-wave signal, we find that the new approach is superior with respect to mass conservation and it allows a much better tracking of outward moving material. This will allow a more accurate simulation of the ejected material and supports the interpretation of present and future multi-messenger observations with more accurate numerical relativity simulations.
\end{abstract}

\section{INTRODUCTION}

Binary neutron star (BNS) systems are a unique laboratory to answer some of the most interesting questions in modern physics. For example: What is the equation of state (EOS) of supranuclear dense matter 1 9]? What is the expansion rate of the Universe [10 14]? How have the heavy elements in the Cosmos 1519 been produced? And, is General Relativity the correct theory to describe gravity 20 23?

An investigation of the full BNS coalescence requires a detailed analysis and understanding of the merger process. Due to the strong gravitational fields and the high velocities of the stars just before merger, one has to solve Einstein's Equations with all nonlinearities using full $3+1 \mathrm{D}$ numerical-relativity simulations [24 26 . Thus, numerical relativity has consolidated its role for the interpretation of compact binary mergers and was used to study the BNS merger GW170817 [27] and its electromagnetic counterparts [28].

To enable stable simulations, state-of-the-art numerical relativity simulations of neutron stars use an artificial atmosphere to model vacuum and near-vacuum conditions outside the stars, see e.g. 26, 29,31. Starting with initial data for neutron stars in vacuum, the standard method fills all the vacuum regions with a very lowdensity atmosphere (with often a cold equation of state). This atmosphere is not physical and artificially added for numerical reasons. One reason for this approach is that for the matter evolution we use conserved matter variables, i.e. variables whose change inside a given cell volume is determined by fluxes across the cell surfaces. To compute these fluxes one has to use interpolation from the cell centers to cell interfaces. In low density regions this interpolation can return matter densities or energies that lie outside what is physically reasonable or allowed. An artificial atmosphere cures these issues. However, even with an artificial atmosphere, some of the same problems can still occur. In addition, the atmosphere has to be tuned to avoid most of these problems, while at the same time keeping it tenuous enough to not unduly influence the simulation. One of the most sophisticated atmosphere implementations is explained in 32 . In this approach a positivity preserving limiter is used for the density. Yet even in this approach a low density atmosphere is still needed. However, it has the advantage that the density of the atmosphere can be made much lower than in more straightforward approaches, so that the effects of the artificial atmosphere can be reduced. There is also a new hydrodynamics approach that uses HamiltonJacobi methods [33, and thus its evolution equations take a different form. So far it has been only used for barotropic fluids, and interestingly for us, in its current formulation it also requires an artificial atmosphere.

Our goal here is to find a scheme that does not explicitly add such an atmosphere. We will first describe the ingredients that allow us to perform simulations that contain true vacuum. After this we discuss tests of our new scheme, where we evolve neutron stars with and without atmosphere.

Throughout this study, we use dimensionless units where $G=c=M_{\odot}=1$. and adopt the signature $(-,+,+,+)$ for the 4-metric. Greek indices on tensors run from 0 to 3 , Latin indices from 1 to 3 , with the standard summation convention for repeated indices. The following can be used to convert from dimensionless units to SI units: time $1000=4.93 \mathrm{~ms}$, distance $1=$ $1.47735 \mathrm{~km}$, energy $1=1.7872 \cdot 10^{47} \mathrm{~J}$ and density $1=$ $6.177413 \cdot 10^{17} \mathrm{~g} / \mathrm{cm}^{3}$

\section{NUMERICAL METHOD}

\section{A. The BAM code}

We perform our dynamical simulations with the BAM code [30, 34 37, which uses the method-of-lines with Runge-Kutta time integrators and finite differences ap- 
proximating spatial derivatives. A Courant-FriedrichLewy factor of 0.25 is employed for all runs (see [34, 38, ).

The numerical domain contains a mesh made of a hierarchy of cell-centered nested Cartesian boxes and consists of $L$ refinement levels from $l=0$ to $L-1$. Each refinement level is made out of one or more equally spaced Cartesian grids with grid spacing $h_{l}$. There are $n$ points per direction on each grid plus a certain number of buffer points on each side. The levels are refined in resolutions by a factor of two such that the grid spacing in level $l$ is $h_{l}=h_{0} / 2^{l}$, where $h_{0}$ is the grid spacing of the coarsest level. The coordinate extent of a grid at level $l \geq 0$ entirely contains grids at any level greater or equal to $l+1$. The moving boxes technique is used to dynamically move and adapt some of the mesh refinement levels during the time evolution. These moving refinement levels are used for the cases like BNS where the center of each star moves during the time evolution. All levels with $l>l_{\mathrm{m}}$ are moving refinement levels. This is implemented in such a way that the moving refinement levels always stay within the coarsest level. The number of points in one direction for moving level $\left(n_{\mathrm{m}}\right)$ can be set to a different value than $n$. There are six buffer points per direction on each sides of refinement grid; cf. Refs. 34, 39 for more information about the buffer points. For simplicity, we always quote grid sizes without buffer points. For the wave zone, a shell made up from six "cubed sphere" patches 40 42 can be added. This helps to improve the accuracy in GW extraction and allows the implementation boundary conditions derived for spherical geometries, see e.g., 43].

\section{B. Spacetime and Matter Evolution}

We employ the Z4c formulation of the Einstein Equations 43 45. combined with the moving puncture gauge using the $1+\log$-slicing condition 46 and the Gamma driver shift [47, 48. For our single star evolutions, Sommerfeld boundary conditions [49] are used. For binary neutron stars, we add spherical patches outside of the coarsest cubic box to allow the use of constraintpreserving boundary conditions 44.

We assume that the matter is properly described as a perfect fluid for which the stress-energy tensor is given by

$$
T^{\mu \nu}=(e+P) u^{\mu} u^{\nu}+P g^{\mu \nu},
$$

with the energy density $e$, the pressure $P$, and the fourvelocity $u^{\mu}$. The total energy density is given by $e=$ $\rho(1+\epsilon)$, where $\rho$ is the rest-mass energy density and $\epsilon$ is the specific internal energy. In many equations we also use the specific enthalpy given by

$$
h \equiv 1+\epsilon+P / \rho .
$$

The matter equations follow from the conservation law for the energy-momentum tensor and the conservation law for the baryon number. Following [50] the equations governing the evolution of general relativistic fluids, can be written in first-order flux-conservative form

$$
\partial_{t} \vec{q}+\partial_{i} \vec{f}^{(i)}(\vec{q})=\vec{s}(\vec{q})
$$

with $\vec{q}$ denoting the conserved variables, $\vec{f}^{(i)}$ the fluxes, and $\vec{s}(\vec{q})$ the source terms. The conserved variables are rest-mass density $(D)$, the momentum density $\left(S_{i}\right)$, and internal energy $(\tau)$ as seen by Eulerian observers. The conserved variables are related to the original variables via

$$
\begin{aligned}
D & =\rho W \\
S_{i} & =\rho h W^{2} v_{i} \\
\tau & =\rho h W^{2}-P-\rho W,
\end{aligned}
$$

where $v_{i}$ is the 3 -velocity and $W$ the Lorentz factor of the fluid.

To close the evolution system, we have to specify an EOS for the fluid. We choose to employ a simple ideal gas EOS in our single star evolutions and a piecewise polytropic fit of the zero-temperature SLy EOS [51, 52] for which we add an additional ideal gas thermal contribution [53] with $\Gamma^{\text {hot }}=1.75$ 54].

\section{Dealing with low density or vacuum regions}

\section{Original implementation in $B A M$}

NSs surrounded by vacuum are modeled in numerical relativity simulations by using an artificial atmosphere, e.g., [30, 55, 57. The artificial atmosphere outside of the stars is chosen as a fraction of the initial central density of the star as $\rho_{\text {atm }} \equiv f_{\text {atm }} \cdot \rho_{c}(t=0)$. The atmosphere pressure and internal energy is computed by employing the zero-temperature part of the EOS. The fluid velocity within the atmosphere is set to zero. At the start of the simulation, the atmosphere is added before the first evolution step. During the recovery of the primitive variables from the conservative variables, a point is set to atmosphere if the density is below the threshold $\rho_{\text {thr }} \equiv f_{\text {thr }} \rho_{\text {atm }}$. In this article, we are using $f_{\text {atm }}=10^{-11}$ and $f_{\mathrm{thr}}=10^{2}$ in all test cases.

\section{A new vacuum treatment}

Conservative to primitive conversion: Unfortunately, in general there is no closed analytic expression for the primitive variables in terms of the conserved ones. We thus have to resort to a root finder. Within our new vacuum treatment, we use the following scheme. We square Eq. (5) and use the definition of the conservative variables to find

$$
W^{2}=\frac{\left(D+\tau+P^{*}\right)^{2}}{\left(D+\tau+P^{*}\right)^{2}-S^{2}} .
$$


Here, $P^{*}$ is an initial guess for the pressure and we have defined $S^{2}=\gamma^{i j} S_{i} S_{j}$. Once $W\left(P^{*}\right)$ is known, we can solve Eqs. (4) and (6) for $\rho$ and $\epsilon$. We obtain

$$
\rho\left(P^{*}\right)=\frac{D}{W\left(P^{*}\right)}
$$

and

$$
\epsilon\left(P^{*}\right)=\frac{\sqrt{\left(D+\tau+P^{*}\right)^{2}-S^{2}}-W\left(P^{*}\right) P^{*}}{D}-1 .
$$

Using a one dimensional root finder, we adjust $P^{*}$ until the EOS of the form $P=P(\rho, \epsilon)$ is satisfied. However, both $W\left(P^{*}\right)$ and $\epsilon\left(P^{*}\right)$ contain a square root of $(D+\tau+$ $\left.P^{*}\right)^{2}-S^{2}$. Thus we need $P^{*}>S-D-\tau$. Furthermore we expect the pressure to be positive. Thus, we need a root finder that searches for the root $P^{*}$ in the interval $\left[P_{\text {min }}, \infty\right)$, where

$$
P_{\min }=\min (0, S-D-\tau) .
$$

Our algorithm employs a Newton-Raphson scheme, but falls back on bisection whenever the Newton step would bring us outside the allowed interval. In addition, we limit Eq. 9 to not violate the weak energy condition, i.e. whenever $\epsilon\left(P^{*}\right) \leq-1$, we set it to $-\left(1 .-10^{-10}\right)$. In most cases we can then find a root and obtain a suitable $P^{*}$. In case where this is not possible, we reset all variables to vacuum, i.e. we set

$$
D=\tau=S_{i}=\rho=\epsilon=P=v_{i}=0 .
$$

We also reset all variables to vacuum if we find that $D<0$, since negative rest mass densities are nonphysical. We point out that similar checks are also present with artificial atmospheres, but with larger threshold values.

Reconstruction and fluxes: The evolution equations for the conserved fluid variables are computed from fluxes at cell interfaces where we do not have grid points. In order to obtain these fluxes, we interpolate the quantities necessary, to compute them at the cell interface locations. For the smooth gravitational fields such as $\alpha$, $\beta^{i}$, and $\gamma_{i j}$ we use sixth order Lagrangian interpolation, while for the potentially non-smooth matter fields we use 5 th order WENOZ interpolation for the primitive variables [36, 58. The interpolation results at each interface are constructed in two ways: once from data to the left $(\mathrm{L})$ of the interface and again from data to the right $(\mathrm{R})$ of the interface. For the primitive variables this results in $\rho_{L / R}, \epsilon_{L / R}$ and $W v_{i} L / R$. Interpolation can still lead to unphysical values on either side. If the determinant of $\gamma_{i j}$ is less than or equal to zero, we set it to 1 and also

\footnotetext{
1 Notice that we interpolate $W v_{i}$ and not $v_{i}$ to avoid cases where
} the 3-velocity is interpolated to a value above light speed. set $\rho_{L / R}=\epsilon_{L / R}=0$. Furthermore, if $\rho_{L}<0$ we set $\rho_{L}=\epsilon_{L}=0$ and if $\rho_{R}<0$ we set $\rho_{R}=\epsilon_{R}=0$. In order to obtain the pressure $P_{L / R}$ as well as the sound speed squared $c_{s L / R}^{2}=\frac{1}{h}\left(\frac{\partial P}{\partial \rho}+\frac{P}{\rho^{2}} \frac{\partial P}{\partial \epsilon}\right)_{L / R}$ we use the EOS. If $c_{s}^{2}<0$ or $c_{s}^{2}>1$ we set it to zero, we also set it to zero if $\rho=0$ or $h=0$. We use the thus interpolated and limited primitive variables to compute the conserved variables as well as the fluxes $\vec{f}_{L / R}$ at both interfaces. In addition, we compute the speeds $\vec{\lambda}_{L / R}$ of the characteristic variables on both sides using

$$
\begin{aligned}
& \lambda_{1}=\alpha \frac{v^{n}\left(1-c_{s}^{2}\right)+\sqrt{C^{2}}}{1-v^{2} c_{s}^{2}}-\beta^{n} \\
& \lambda_{2}=\alpha \frac{v^{n}\left(1-c_{s}^{2}\right)-\sqrt{C^{2}}}{1-v^{2} c_{s}^{2}}-\beta^{n} \\
& \lambda_{3}=\alpha v^{n}-\beta^{n} \\
& \lambda_{4}=\alpha v^{n}-\beta^{n} \\
& \lambda_{5}=\alpha v^{n}-\beta^{n}
\end{aligned}
$$

where $C^{2}=c_{s}^{2}\left\{\left(1-v^{2}\right)\left[g^{n n}\left(1-v^{2} c_{s}^{2}\right)-v^{n} v^{n}\left(1-c_{s}^{2}\right)\right]\right\}$, $v^{n}=v^{i} n_{i}$ and $n_{i}$ is the normal to the interface. If $1-$ $v^{2} c_{s}^{2}=0$ or $C^{2}<0$ we simply set $\lambda_{1}=\lambda_{2}=0$.

The final numerical flux $\vec{F}$ at the interface is then computed using a standard method such as the Local LaxFriedrichs (LLF) scheme where

$$
\vec{F}_{\text {interface }}=\frac{1}{2}\left[\vec{f}_{R}+\vec{f}_{L}-|\lambda|_{\max }\left(\vec{q}_{R}-\vec{q}_{L}\right)\right] .
$$

Here $\vec{q}_{L / R}$ are the conserved variables on the left or right and $|\lambda|_{\max }$ is the characteristic speed with the largest magnitude. In fact, in the simulations presented in this paper we use the LLF flux always at low densities, while possibly using a higher order flux at higher densities. In this case, the higher-order flux $\vec{F}_{\text {interface }}^{H O}$ is obtained by interpolating the characteristic variables from five neighboring points using the WENOZ scheme [36]. In some simulations labeled with $\mathrm{HO}$ we use this higher

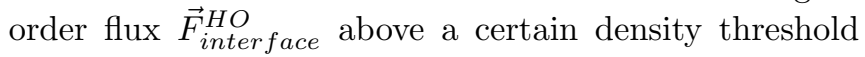
(typically on the order of $1 \%$ of the maximum of $\rho$ at the star center).

Matter removal: We use a forth order Runge-Kutta scheme to evolve the conserved variables. Before we evaluate the right hand side within each Runge-Kutta substep, we set the conserved variables to vacuum if one of the following two conditions is true: (1) if $D<0$, (2) if $D>f W \rho$ and $\alpha<0.2$, where the factor $f$ is usually chosen to be 100 . The first condition is obvious and allows only positive matter density. The second case is used for matter removal inside black holes (BHs). Since we use the standard moving puncture gauges $1+\log$-lapse and gamma-driver shift [47, 48, 59, the $\mathrm{BH}$ horizon is located near the surface where $\alpha \sim 0.3$. Therefore, condition (2) is true only inside the horizon. We have observed that when matter accumulates near the $\mathrm{BH}$ center after a star 
TABLE I: Parameters and properties of the single neutron star tests. We report the density at which we switch between primitive and characteristic reconstruction $\left(\rho_{\text {switch }}\right)$, threshold density and atmosphere density for the artificial atmosphere $\left(\rho_{\text {thr }} \& \rho_{\text {atm }}\right)$, EOS parameters $\Gamma$ and $K$ for the polytropic $\operatorname{EOS}\left(p=K \rho^{\Gamma}\right)$ to setup the initial data, and employed symmetry to reduce computational costs. For completeness, we also present the gravitational mass $M$, the baryonic restmass $M_{b}$, the initial central density $\rho_{c}$, the equatorial radius $R_{e}$, and the aspect ratio $R_{p} / R_{e}$.

\begin{tabular}{|l|c|c|c|}
\hline Stars & TOV $_{\text {static }}$ & $\mathrm{TOV}_{\text {mig }}$ & $\mathrm{RNS}_{\text {col }}$ \\
\hline$\rho_{\text {switch }} \cdot 10^{-5}$ & 1.28 & 7.83 & 3.12 \\
$\rho_{\text {thr }} \cdot 10^{-12}$ & 1.280 & 7.9934 & 3.1160 \\
$\rho_{\text {atm }} \cdot 10^{-14}$ & 1.280 & 7.9934 & 3.1160 \\
$\Gamma$ & 2 & 2 & 2 \\
$K$ & 100 & 100 & 99.5 \\
Symmetry & octant & octant & quadrant \\
\hline$M$ & 1.400 & 1.448 & 1.861 \\
$M_{b}$ & 1.506 & 1.535 & 2.044 \\
$\rho_{c} \cdot 10^{-3}$ & 1.2800 & 7.9934 & 3.1160 \\
$R_{e}$ & 8.126 & 4.268 & 9.652 \\
$R_{p} / R_{e}$ & 1 & 1 & 0.65 \\
\hline
\end{tabular}

collapses, $D$ rises much faster than $\rho$ so that Eq. (4) is violated. This happens because the $\mathrm{BH}$ center in this gauge is only very poorly resolved 60, 61. Condition (2) ensures that matter is removed whenever $D$ becomes much larger than $\rho$. An alternative approach was described in 62, where matter is not removed, but some of the eigenvalues and $W$ are reset for regularity, which could be explored in future work.

\section{SINGLE STAR SPACETIMES}

To test our new implementation, we start by studying three different single star configurations:

1. a stationary, static star $\left(\mathrm{TOV}_{\text {static }}-\mathrm{Sec}\right.$ III A

2. an unstable, migrating star $\left(\mathrm{TOV}_{\mathrm{mig}}-\mathrm{Sec}\right.$. III B

3. a perturbed, collapsing, uniformly rotating neutron star $\left(\mathrm{RNS}_{\mathrm{col}}-\mathrm{Sec}\right.$. III C)

Each test uses the LLF flux with primitive reconstruction 63 65, labeled as LLF, and the hybrid scheme employing characteristic reconstruction for large and primitive reconstruction for low densities (labeled as HOLLF). We employ four different resolutions denoted as Low, Mid, High, and Fine. Details about the physical setup and the grid parameters are given in Tab. I and Tab. II. respectively. In order to assess the performance of the old 'atmosphere' and the new 'vacuum' method, we compare the central density, the total rest-mass, and the Hamiltonian Constraint during the evolution for all tests.
TABLE II: The grid parameters for the single star configurations at all four resolutions are tabulated here. The atmosphere and vacuum treatments have the same parameters and thus are tabulated only once. $L$ is the total number of boxes, $n\left(n^{m v}\right)$ is the number of points in the fixed (moving) boxes, and $h_{0}, h_{L-1}$ are the grid spacing in level $l=0, L-1$. The grid spacing in level $l$ is $h_{l}=h_{0} / 2^{l}$.

\begin{tabular}{|c|cccccc|}
\hline & \multicolumn{6}{|c|}{ Grid Parameters } \\
\hline Tests & Resolutions & $L$ & $n$ & $n^{m v}$ & $h_{0}$ & $h_{L-1}$ \\
\hline \multirow{5}{*}{ TOV $_{\text {static }}$} & Low(L) & 5 & 64 & 64 & 1.125 & 0.281 \\
& $\operatorname{Med}(\mathrm{M})$ & 5 & 96 & 96 & 0.750 & 0.188 \\
& High(H) & 5 & 128 & 128 & 0.563 & 0.141 \\
& Fine(F) & 5 & 160 & 160 & 0.450 & 0.113 \\
\hline \multirow{5}{*}{ TOV $_{\text {mig }}$} & Low(L) & 7 & 64 & 64 & 19.20 & 0.300 \\
& Med(M) & 7 & 96 & 96 & 12.80 & 0.200 \\
& High(H) & 7 & 128 & 128 & 9.600 & 0.150 \\
& Fine(F) & 7 & 160 & 160 & 7.680 & 0.120 \\
\hline \multirow{5}{*}{ RNS $_{\text {col }}$} & Low(L) & 9 & 64 & 28 & 18.00 & 0.070 \\
& Med(M) & 9 & 96 & 42 & 12.00 & 0.047 \\
& High(H) & 9 & 128 & 56 & 9.00 & 0.035 \\
& Fine(F) & 9 & 160 & 72 & 7.20 & 0.028 \\
\hline
\end{tabular}

\section{A. Stationary TOV simulations}

In Fig. 1, we plot the relative central density $1-\frac{\rho_{c}(t)}{\rho_{c}(t=0)}$, the relative rest-mass change $\left|1-\frac{M_{b}(t)}{M_{b}(t=0)}\right|$, and the Hamiltonian constraint for all $\mathrm{TOV}_{\text {static }}$ simulations. All quantities are extracted at level $l=4$ which is the finest level, but also fully covers the entire star. The stars are evolved up to a time of $1000 M_{\odot}$, i.e., $4.93 \mathrm{~ms}$. Truncation errors trigger small-amplitude pulsations in the stars [55, 66 that can be seen as oscillations in the relative central density. The central density oscillations are larger for the hybrid HOLLF method, but decrease clearly with an increasing resolution. There is no noticeable difference between the old atmosphere and the new vacuum method.

Considering the mass conservation, one sees a clear advantage of our new implementation. In the case of the old atmosphere method, the limit for setting the density to the atmosphere value is $1.28 \cdot 10^{-12}$. In the first few time steps, the star surface slightly grows causing the density to drop below this threshold. This leads to a visible violation of mass conservation after the first timestep. With our new vacuum approach a low density layer builds up around the star. Thus with our new approach mass is much better conserved. In addition, even during the subsequent evolution one observes a larger mass violation for the atmosphere method than for our new implementation, where for the highest resolution the mass violation is below $10^{-8}$.

It is also important to point out that for the atmosphere case, we do not observe convergence in the mass. This applies to both the HOLLF and the LLF scheme. 


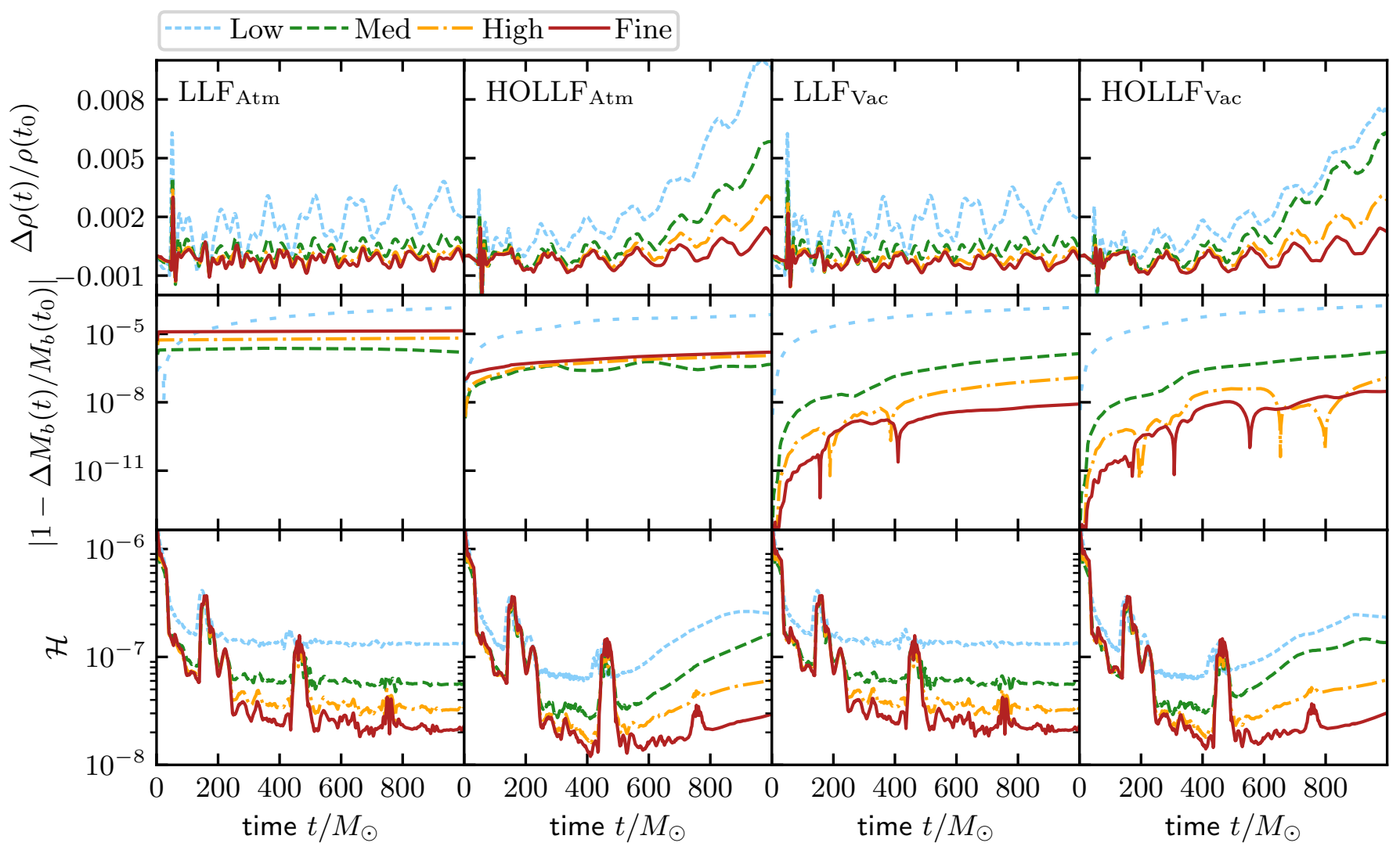

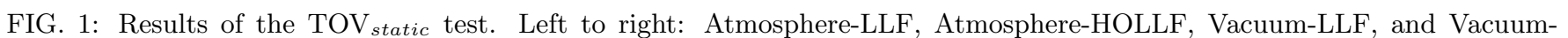
HOLLF. Top: Relative change in central density $1-\frac{\rho_{c}(t)}{\rho_{c}(t=0)}$. Middle: Relative rest-mass change $\left|1-\frac{M_{b}(t)}{M_{b}(t=0)}\right|$. Bottom: The time evolution of Hamiltonian Constraint $(\mathcal{H})$.

On the contrary, for the new vacuum method we find second order convergence for most of the time for LLF and up to $t=600 M_{\odot}$ for HOLLF.

The bottom panel of Fig. 1 shows the evolution of the Hamiltonian constraint. We observe a reduction of the Hamiltonian constraint for increasing resolution exhibiting clean second-order convergence. As an example we show a convergence test for the LLF vacuum setup in Fig. 2. Here the differences in the Hamiltonian Constraint are scaled by factors that correspond to assuming second order convergence. These scaled lines nicely coincide with the middle line as expected for second order convergence.

\section{B. Migration of an unstable star}

TOV $_{\text {mig }}$ represents a test based on the unstable U0 model of Ref. [30, with a central energy density of $\epsilon_{c}=8.73 \times 10^{-3}$ and a gravitational mass of $M=1.557$, A small perturbation caused by truncation errors leads to pulsations that migrate the star towards a stable configuration of the same rest-mass. Initially, the central density decreases and the star expands rapidly. Later its inner core contracts which leads to a shrinking of the

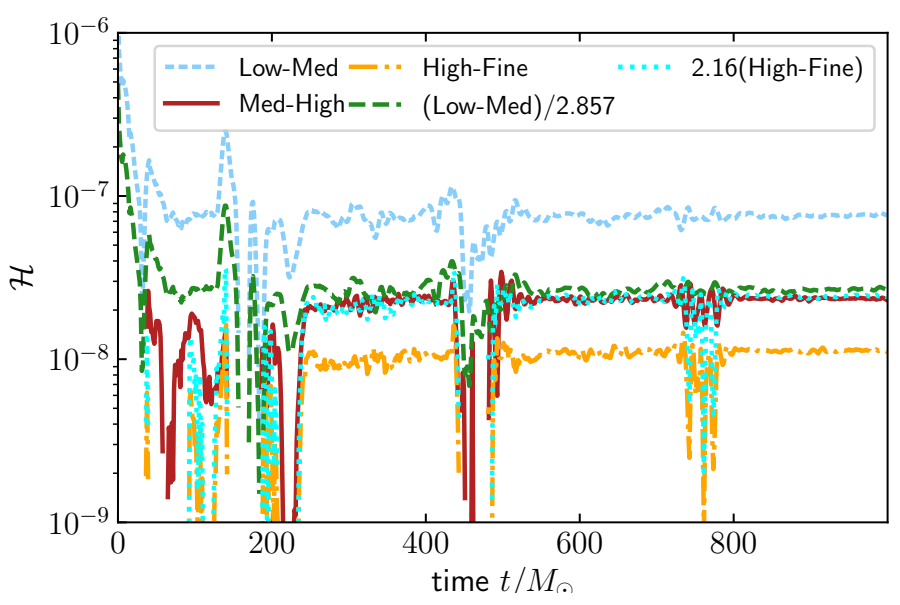

FIG. 2: Convergence test of Hamiltonian Constraint of LLF

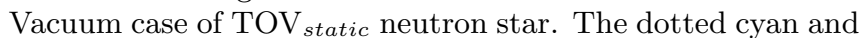
dashed green lines are obtained by scaling the dash-dotted orange line and the dashed blue line respectively in order to match up with the solid red line.

star and an increase of the central density. As a result, it pulsates causing matter to cross the grid refinement boundaries. To better resolve the dynamics we are using 


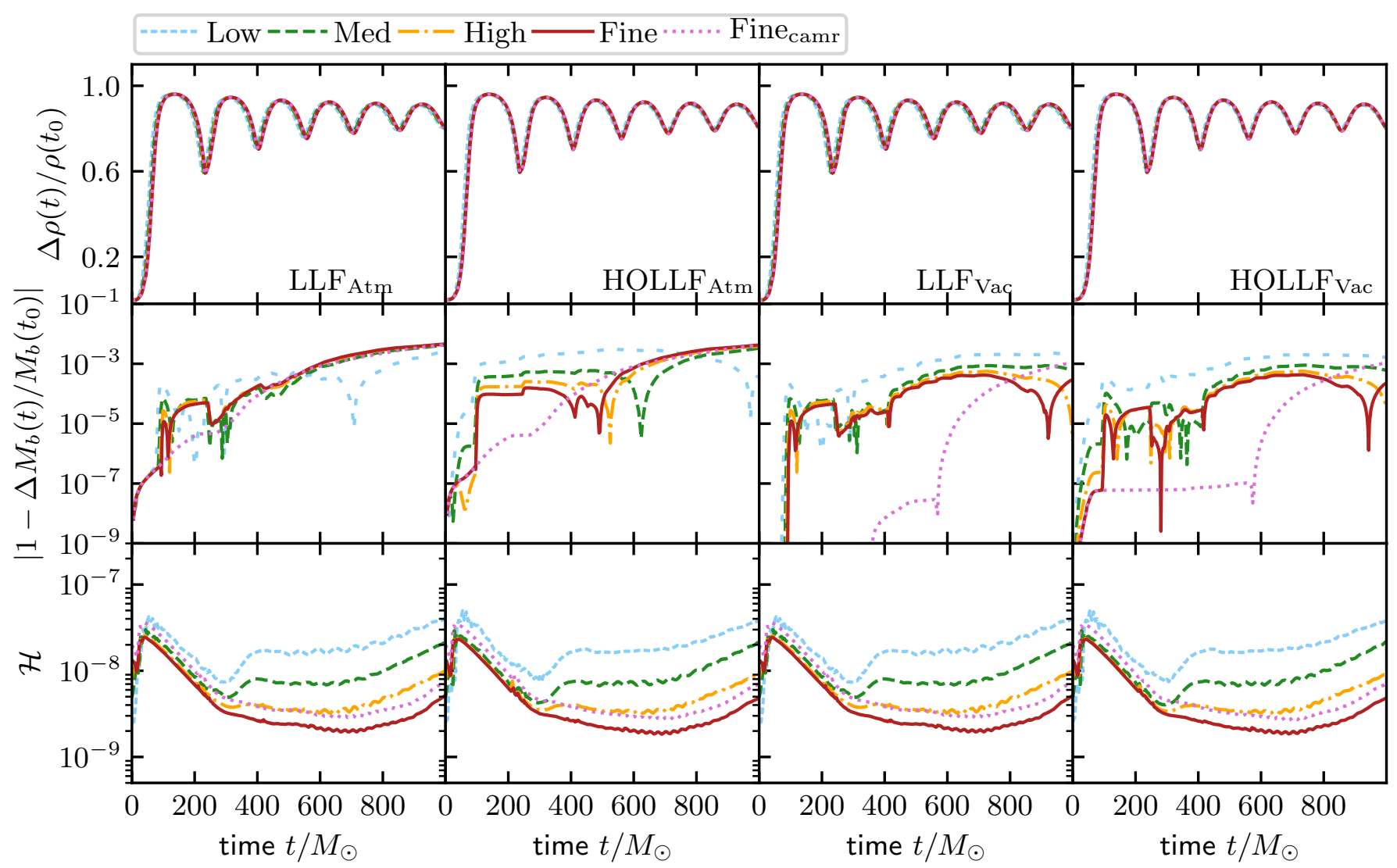

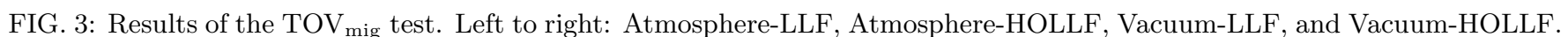
Top: Relative change in central density $1-\frac{\rho_{c}(t)}{\rho_{c}(t=0)}$. Middle: Relative rest-mass change $\left|1-\frac{M_{b}(t)}{M_{b}(t=0)}\right|$. Bottom: The time evolution of Hamiltonian Constraint $(\mathcal{H})$. Since matter is expected to cross refinement boundaries during this test, we also perform for the highest resolution, a simulation in which we apply the conservative refluxing algorithm that we developed in [35].

a larger number of refinement levels than in the $\mathrm{TOV}_{\text {static }}$ test.

In Fig. 3 we plot the central density on the finest level $l=6$ and the rest-mass and Hamiltonian constraint on level $l=1$. In the top panels, we see a decrease in the amplitude of pulsation of central density as the simulation progresses. If we would run the simulation longer, the star would finally settle down to a stable configuration. The Hamiltonian constraint in the bottom panel converges roughly with a second-order in all four cases. For the LLF case we see convergence throughout the simulation whereas in HOLLF we see convergence roughly from $300 M_{\odot}$ to $1000 M_{\odot}$. Thus considering the Hamiltonian constraint, the LLF simulations perform better than the HOLLF ones.

As for $\mathrm{TOV}_{\text {static }}$ case, we find a better mass conservation for the vacuum configurations than for the old atmosphere method. Convergence consistent with the second-order is observed in the early part of the simulations HOLLF simulations, for the LLF method no convergence is present at all. During the evolution time and because of the pulsation of the star, mass is crossing the refinement level. At this time, mass conservation is generally lost if no additional conservative refluxing step as introduced in [35, 67] is applied. To prove this point, we perform a simulation with the highest resolution and activate the refluxing scheme, labeled as Fine ${ }_{\text {camr }}$ in Fig. 3. We find that for the Vacuum method mass conservation is significantly improved up to about $t=600 M_{\odot}$. At this time low density material hits the outer boundary of the considered computational domain and leaves it, consequently the total mass can not be conserved after this point.

Considering 2d-snapshots of the matter evolution clearly reveals the advantage of the new vacuum treatment. As can be seen in Fig. 4, when very low density material expands, it is stopped due to the artificial atmosphere (see bottom left panel) while it expands freely in the vacuum case (bottom right panel). Such an artificial impact on the outgoing matter could be of significant importance if one wants to track outward going ejecta.

\section{Collapsing, rotating neutron star}

As a last single-star test case, we study the collapse of a rotating neutron star (RNS). This test aims towards a better understanding if a $\mathrm{BH}$ can be properly modeled 

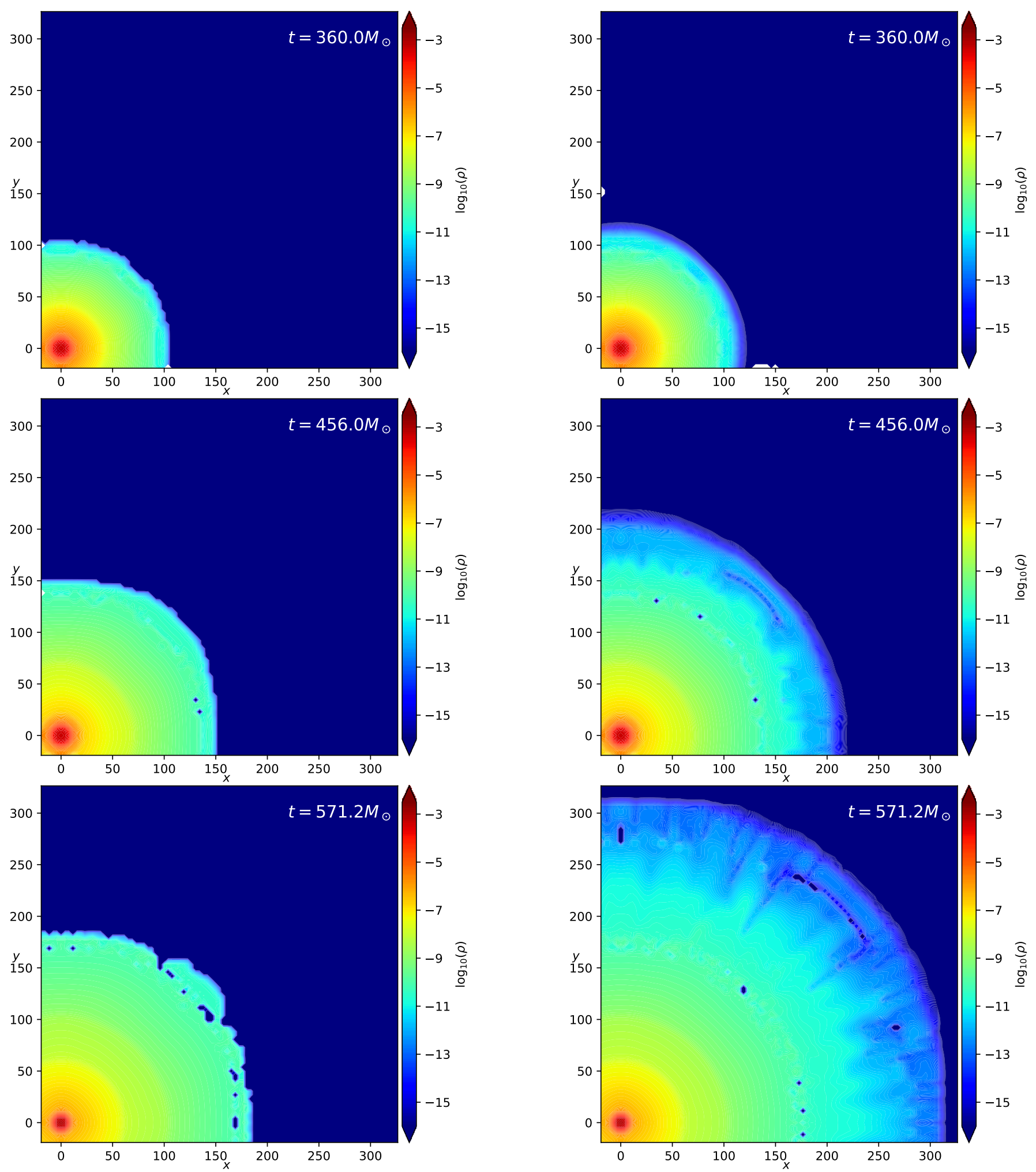

FIG. 4: The rest-mass density in xy-plane for the TOV $\mathrm{Tig}_{\text {mig }}$ test on refinement level $l=1$ at different times for the finest resolution ( $\mathrm{n}=160$ points) employing the conservative mesh refinement [35. The left panels show the previous atmosphere scheme, the right panel shows the new vacuum implementation. For the atmosphere case, the threshold density below which artificial atmosphere is set up $\rho_{t h r}$ is $\sim 7.9934 \cdot 10^{-12}$ and the artificial atmosphere level $\left(\rho_{a t m}\right)$ is $\sim 7.993 \cdot 10^{-14}$. 


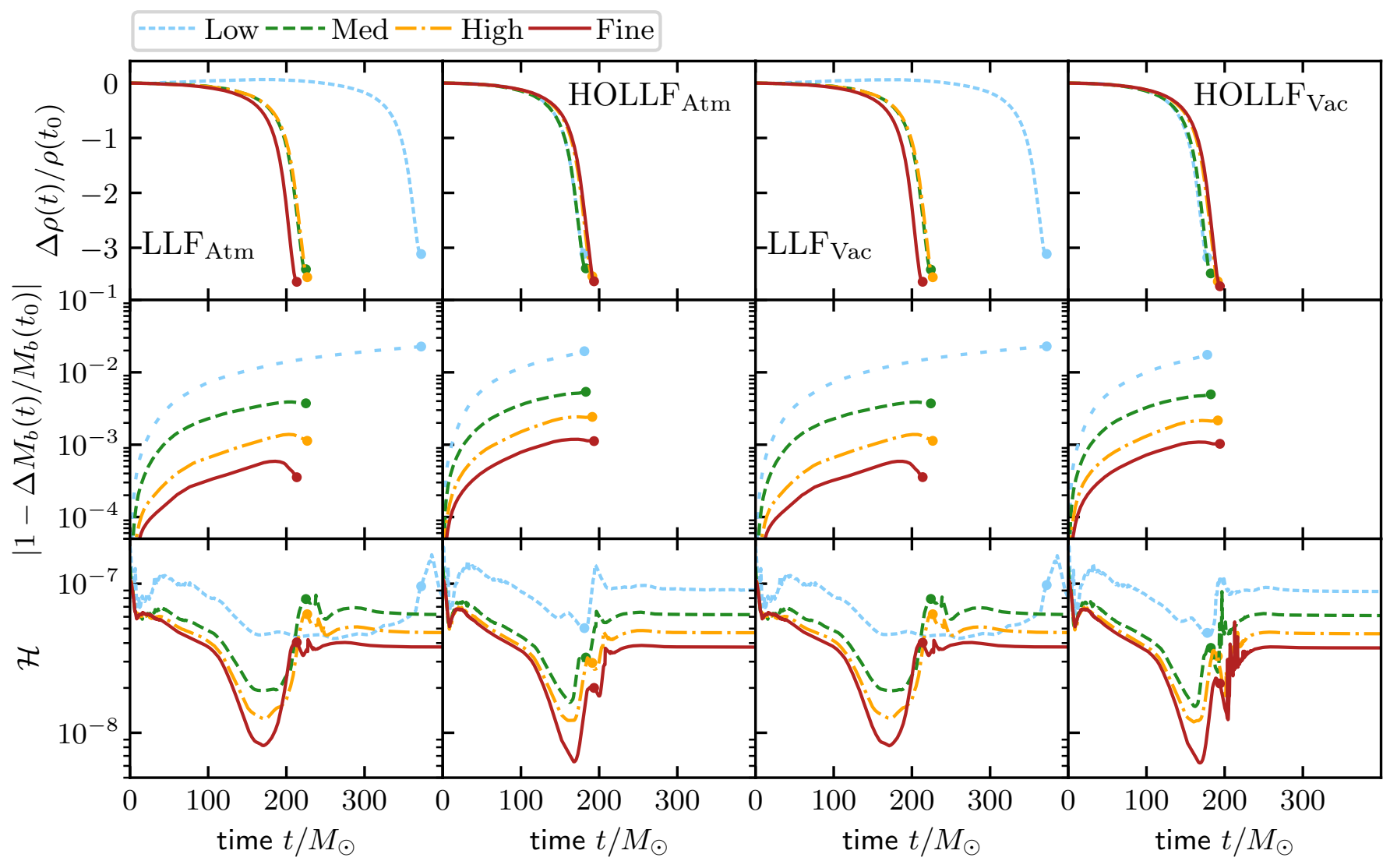

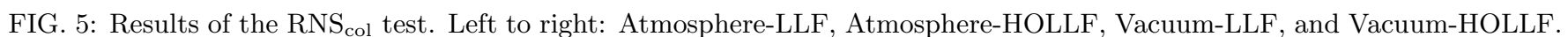
Top: Relative change in central density $1-\frac{\rho_{c}(t)}{\rho_{c}(t=0)}$. Middle: Relative rest-mass change $\left|1-\frac{M_{b}(t)}{M_{b}(t=0)}\right|$. Bottom: The time evolution of Hamiltonian Constraint $(\mathcal{H})$.

within our new algorithm.

The initial data are computed using a polytropic EOS with $K=100, \rho_{c}=3.1160 \cdot 10^{-3}$, and axes ratio 0.65 , which leads to a star with gravitational mass of $M=1.861$, baryonic mass of $M_{b}=2.044$, and an angular velocity of $\Omega=3.96 \times 10^{-2}$. The star is evolved with the polytropic EOS with $K=99.5$ and $\Gamma=2$. This initial perturbation due to the change of the EOS triggers the collapse of the star to a $\mathrm{BH}$. A similar configuration has been investigated in the past, e.g., Refs. 68 70. We are evolving the star with quadrant symmetry, i.e., use reflection symmetry along the $x$ - and $y$-axis and employ nine refinement levels.

In Fig. 5 we plot the central density in the finest level $l=8$ and rest-mass and Hamiltonian constraint on level $l=3$. The collapse to a BH happens at around $t=200 M_{\odot}$ for most cases except for the lowest resolution using the LLF scheme. For both vacuum and atmosphere cases with LLF at $n=64$ points collapse happens at around $t=380 M_{\odot}$. After the star collapses into a $\mathrm{BH}$, matter is removed to avoid the occurrence of steep density gradients as mentioned before.

The Hamiltonian constraint shows second-order convergence before the $\mathrm{BH}$ formation. After the collapse, the convergence order reduces to first order. In both vacuum and atmosphere cases, the error of rest-mass behaves in a similar way.

Overall, we find no clear and noticeable difference between the old atmosphere and new vacuum method.

\section{Summary of the single star simulations}

We have studied evolutions with an updated implementation of our vacuum treatment for a number of single star spacetimes. The main observations are:

- Mass conservation can be improved with the new implementation; cf. $\mathrm{TOV}_{\text {static }}$.

- The new implementation improves the simulation of outflowing, low density material; cf. TOV $\mathrm{Tig}_{\text {; }}$;

- The new vacuum method is capable of tracking the $\mathrm{BH}$ formation; cf. $\mathrm{RNS}_{c o l}$. 
TABLE III: The grid parameters for the BNS simulations. The atmosphere and vacuum simulations use the same grid configurations to allow a proper comparison. $L$ denotes the total number of levels, $l^{m v}$ the finest non-moving level, $n$ $\left(n^{m v}\right)$ the number of points in the fixed (moving) boxes, and $h_{0}, h_{L-1}$ are the grid spacings in level $l=0, L-1$. The grid spacing of level $l$ is $h_{l}=h_{0} / 2^{l}$. $n_{r}$ is the radial point number and $n_{\theta}$ is angular point number.

\begin{tabular}{|c|cccccccc|}
\hline Resolutions & $L$ & $l^{m v}$ & $n$ & $n^{m v}$ & $h_{0}$ & $h_{L-1}$ & $n_{r}$ & $n_{\theta}$ \\
\hline Low & 7 & 2 & 128 & 64 & 15.040 & 0.235 & 128 & 56 \\
Med & 7 & 2 & 192 & 96 & 10.027 & 0.157 & 192 & 84 \\
High & 7 & 2 & 256 & 128 & 7.520 & 0.117 & 256 & 112 \\
Fine & 7 & 2 & 320 & 160 & 6.016 & 0.094 & 320 & 140 \\
\hline
\end{tabular}

\section{BINARY NEUTRON STAR EVOLUTIONS}

\section{A. Binary configurations}

Finally, we want to discuss the performance of our new vacuum treatment for the simulation of BNS setups. We focus here on the simulation of an equalmass, non-spinning configuration evolved with the old atmosphere and the new vacuum method. To save computational costs, we only perform simulations with the HOLLF scheme, for which Ref. [36] showed its superiority compared to the LLF scheme with primitive reconstruction.

The individual stars have a baryonic mass of 1.495 and a gravitational mass in isolation of 1.350. For the EOS, we use a piecewise polytropic fit of the zero-temperature SLy EOS [51, 52, 71] and add an additional thermal idealgas pressure component during the dynamical simulation.

The initial data is calculated by using the pseudospectral SGRID code $72+74$. The initial separation of the stars is 35.5 , i.e., $52.4 \mathrm{~km}$, which results in an orbital frequency of 0.0070 , an initial ADM mass of 2.678, and an initial ADM angular momentum of 7.686. The eccentricity of the inspiral is approximately $1.3 \cdot 10^{-4}$. This relatively low value has been achieved by using the eccentricity reduction discussed in [74].

Details about the grid setup for the BAM evolutions are given in Tab. III. To save computational costs, we have employed bitant symmetry. In contrast to the single-star tests, we substitute the outermost Cartesian box (level $l=0$ ) by a shell made up from six "cubed sphere" patches [40 42]. In this shell matter is not evolved.

\section{B. Dynamical Evolution}

During the inspiral, a general (almost linear) reduction of the central density is visible. This linear trend reduces significantly with increasing resolution and is connected to the numerical dissipation [36], which decreases with decreasing grid spacing; cf. Fig. 6. Overall, there is gen-
TABLE IV: Merger times for atmosphere and vacuum cases at different resolutions. Here Low, Med, High, and Fine are simulation with resolutions $64,96,128$, and 160 points respectively. The merger time values are in geometric units where $6000 M_{\odot} \approx 30 \mathrm{~ms}$.

\begin{tabular}{|l|cccc|}
\hline Tests & Low & Med & High & Fine \\
\hline BNS Atm & $6117 M_{\odot}$ & $6583 M_{\odot}$ & $6682 M_{\odot}$ & $6740 M_{\odot}$ \\
\hline BNS Vac & $6150 M_{\odot}$ & $6612 M_{\odot}$ & $6689 M_{\odot}$ & $6737 M_{\odot}$ \\
\hline
\end{tabular}

erally a second-order convergence in the central density visible for both, the atmosphere and the vacuum method.

In addition, we plot the time evolution for the central density for the two highest resolutions in Fig. 7. Clearly visible are large density oscillations after the merger, which correspond to radial oscillations of the formed hypermassive neutron star (HMNS), see e.g. Refs. [77, 78] for further details. The main difference between the old atmosphere and the new vacuum method is that for the two highest resolutions the lifetime of the HMNS is shorter for the vacuum method than for the atmosphere treatment. We note that the determination of the remnants lifetime does influence (i) the material outflow and its composition and (ii) the properties of the $\mathrm{BH}+$ disk system, i.e., the potential short gamma-ray burst.

The middle panel of Fig. 6 shows the conservation of the rest-mass density, where we note that these simulations do not employ the conservative refluxing algorithm yet. We plan to repeat the simulations with conservative refluxing in the future when we have more computer time. The error of the rest-mass seems to decrease as we go to higher resolutions. For the highest resolution, the total rest-mass in conserved up to $0.5 \%$ throughout the inspiral, independent of the employed atmosphere/vacuum scheme. For the Hamiltonian constraint, convergence consistent with second-order is seen until $2000 M_{\odot}$ in both the atmosphere and vacuum case. After that, the order of the convergence rises up to fourth order which is is higher than is theoretically expected. However, throughout the simulation there is clear pattern of Hamiltonian constraint decreasing for both cases as we go to higher resolutions. Looking at the plots of these three quantities, there is not a clear advantage for either the atmosphere or the vacuum method.

\section{Gravitational Waveforms}

For both methods an increasing resolution leads to a later merger, which is due to the increase of the numerical dissipation for lower resolutions. For simulations with the BAM code this effect is discussed in [36. We report the merger time in Tab. IV for all BNS simulations. Most importantly, the difference between the atmosphere and vacuum method decreases with increasing resolution, consequently, both methods seem to lead to a similar continuum limit. 


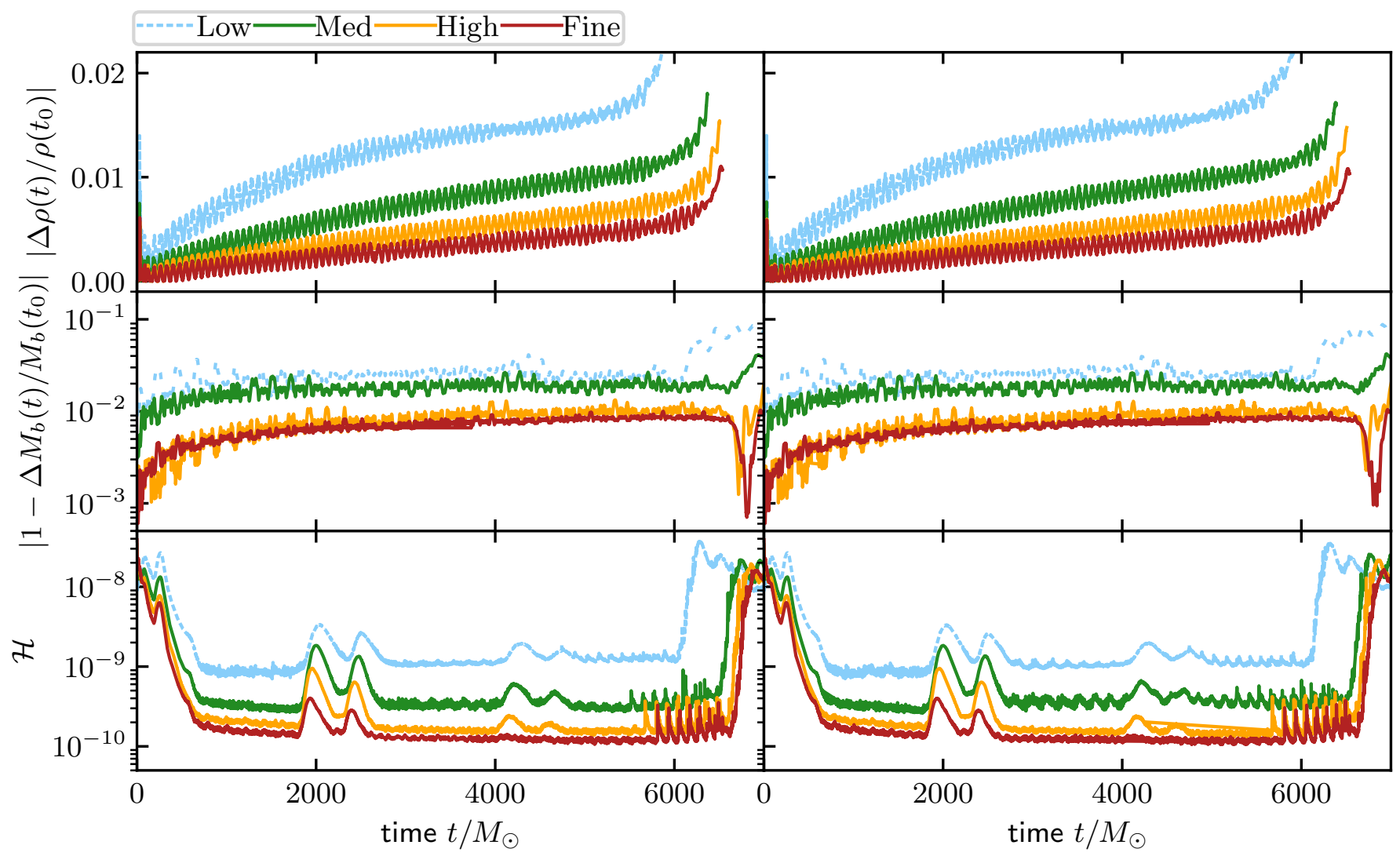

FIG. 6: Results of the BNS runs. Left to right: Atmosphere-HOLLF and Vacuum-HOLLF. Top: Relative change in central density $1-\frac{\rho_{c}(t)}{\rho_{c}(t=0)}$. Middle: Relative rest-mass change $\left|1-\frac{M_{b}(t)}{M_{b}(t=0)}\right|$. Bottom: The time evolution of Hamiltonian Constraint $(\mathcal{H})$. We employ a Savitzky-Golay filter [75, 76, to increase the visibility of the presented curves.

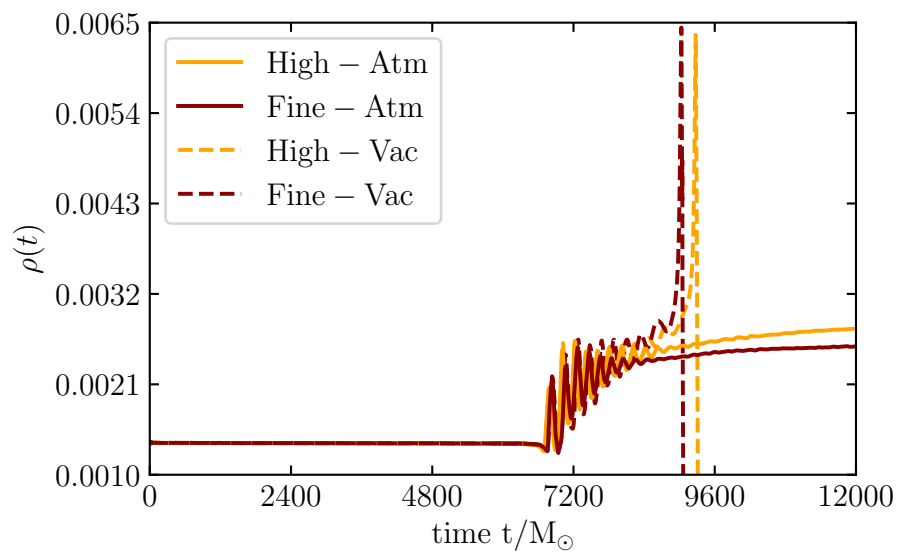

FIG. 7: Central density of the BNS for the simulations with the two highest resolutions. Solid lines are for the atmosphere and the dashed lines are for the vacuum methods. This plot shows how density changes in BNS during inspiral, merger, post-merger, and black hole formation.

In Fig. 8, we present the GW signal for all simulations in the top panels for the atmosphere (left) and the vacuum (right) methods. The bottom panels shows the phase differences between different resolutions, we rescale the phase differences assuming second-order convergence (dashed lines) and find generally that the both methods show the expected convergence order with a sightly better convergence behavior for the original atmosphere treatment. For all methods the Low-resolution simulation stops being second-order convergent after about $1500 M$, which indicates that this resolution is not sufficient to be in the convergent regime until merger. Overall, we find very close agreement between the individual phase differences reported in the Fig. 8. In the right bottom panel, we show the phase difference between the two highest resolutions for the vacuum (solid, orange) and the atmosphere method (purple, dotted). We find that the difference is almost identical, thus, there is no improvement in the extracted GW signal for our new vacuum method, which we assume is caused by the fact that the overall bulk motion is dominating the $\mathrm{GW}$ radiation and that the new vacuum treatment mostly affects the low density regions.

\section{Ejecta Quantities}

Since the amount of ejected material is tightly connected to the creation of electromagnetic counterparts for 


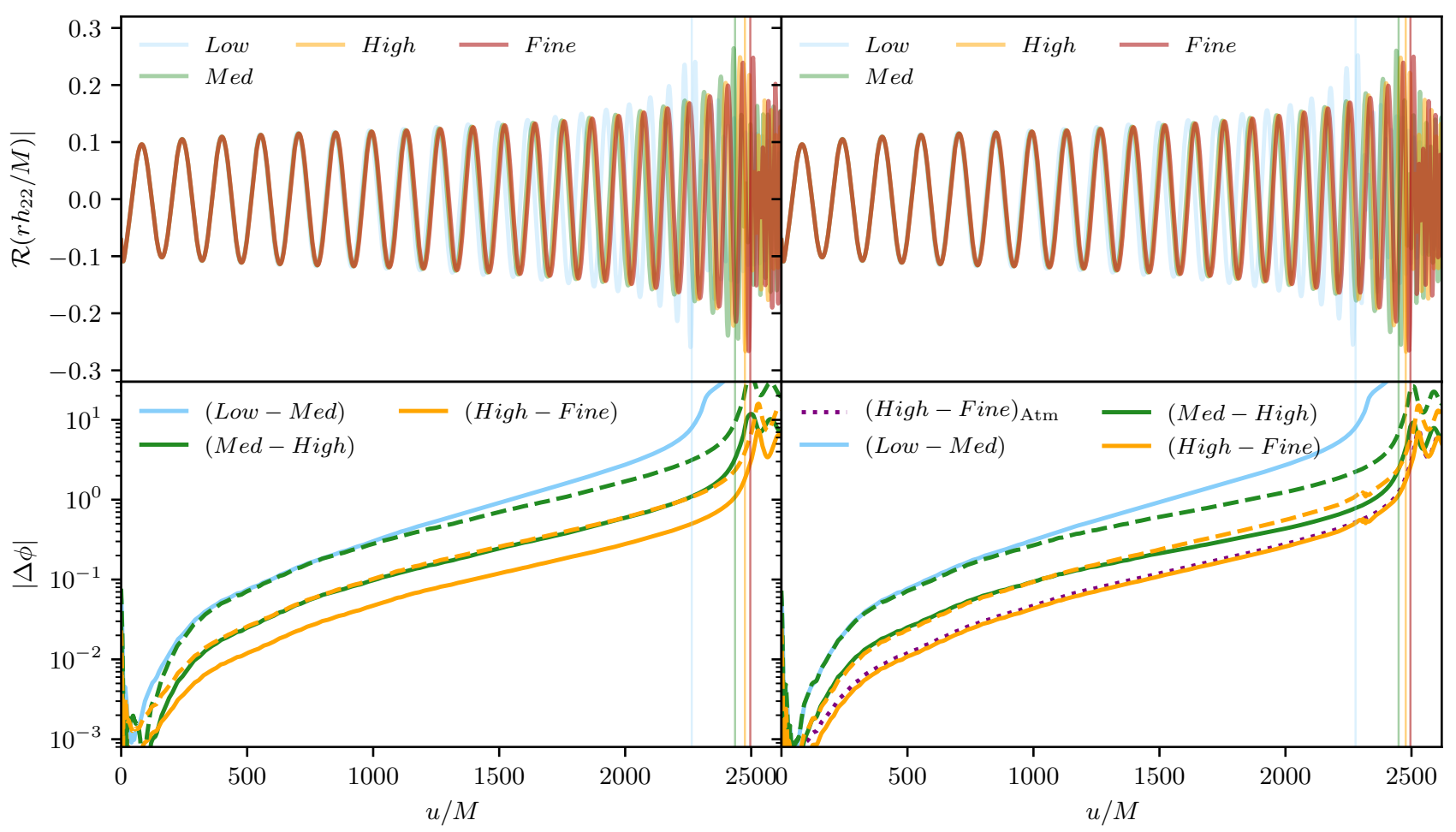

FIG. 8: In the top panels, we plotted the GW signal for both the old atmosphere method (left) and the new vacuum method (right). The bottom panels show the phase differences between different resolutions. The dashed lines are the rescaled phase differences assuming second-order convergence. The bottom right panel includes also the phase difference of the two highest resolutions for the atmosphere method for an easier comparison. Overall, we find similar phase difference and convergence properties for methods.

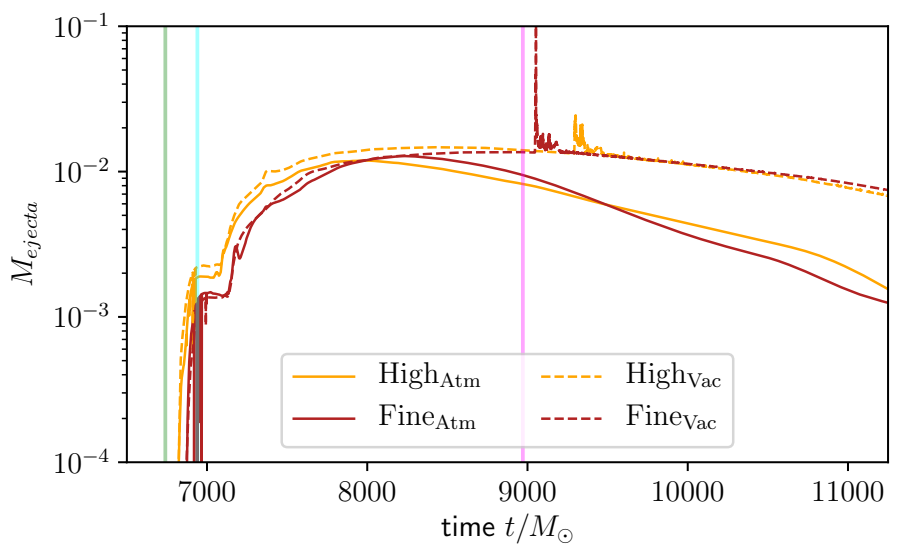

FIG. 9: The ejecta mass for the two highest resolutions. The vertical lines indicate the merger time, the time $1 \mathrm{~ms}$ after the merger, and the time $11 \mathrm{~ms}$ after the merger. For the latter two, 2D plots are shown in Figs. 10 and 11. The spike in the vacuum simulations occurs due to the formation of the $\mathrm{BH}$.

a BNS merger, see e.g. Ref. 79 and references therein, it is important to improve the evolution of low density material.

Generally, when material gets ejected, the fluid expands and the density reduces until it finally falls below the artificial atmosphere threshold used within the at- mosphere implementation. In the atmosphere case such fluid elements are then set to atmosphere values with zero velocity, and are thus no longer counted as ejecta, so that the ejecta mass decreases. This trend is clearly visible in Fig. 9. The problem is mostly present at the outer region of the ejected material for material which moves with the highest velocities. Consequently, removing this material leads also to a drop in $v_{\mathrm{ej}}$ for the atmosphere simulations.

We have made $2 \mathrm{D}$ plots to further investigate the difference in mass ejection in the atmosphere and the vacuum methods (see Figs. 10 and 11). We plot the mass density, velocity, and ejecta mass density at level $l=1$ of the finest resolution simulation. We use a linear (velocity) as well as a logarithmic (mass density and ejecta mass density) color scales and plot two snapshots in time at $1 \mathrm{~ms}$ (Fig. 10) and $11 \mathrm{~ms}$ (Fig. 11) after the merger. We choose these two times for the following reasons. Both methods begin with identical ejecta, same velocity and similar mass density. As the ejecta expand to a larger radius the density in the outer regions of the ejecta mass drop below the atmosphere threshold value. Thus, lowdensity material is removed and the ejected matter never reaches the outer boundary. Therefore, we find that the outflowing material seems to stall about $\sim 10 \mathrm{~ms}$ after the merger at a maximum radial extend of $\sim 350$. In the vacuum case, the ejecta moves further out. The eventual mass reduction for the vacuum method in Fig. 9 at late 

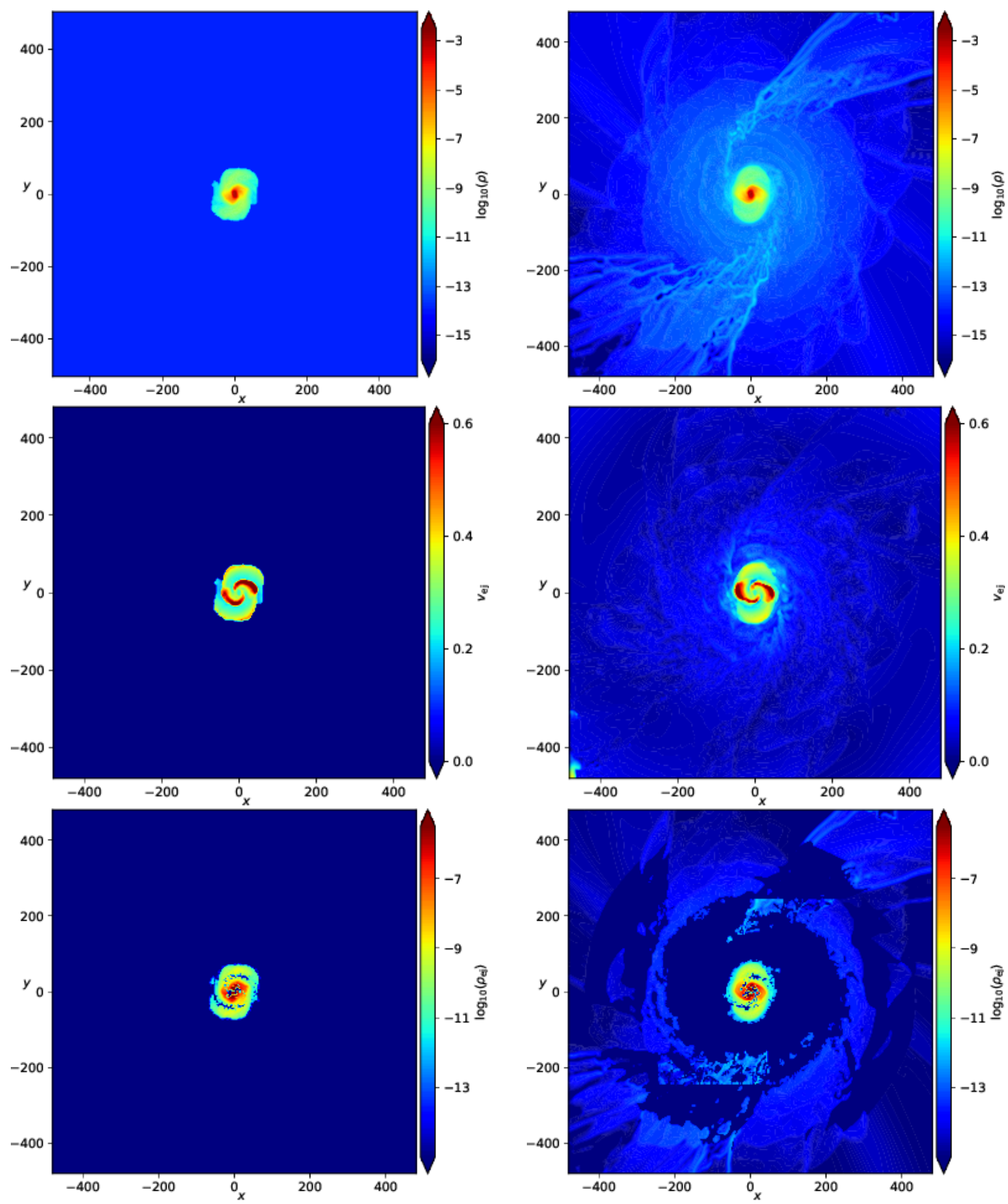

FIG. 10: Snapshots of the mass density (top), velocity (middle), and ejecta mass density (bottom) in xy-plane of BNS simulation at $1 \mathrm{~ms}$ after merger (vertical cyan line in Fig. 9p. The finest resolution for both the atmosphere (left) and the vacuum (right) is plotted with a linear (for velocity) and logarithmic color scales (for mass density and ejecta mass density). This is on level $l=1$, which extends up to 481.28. The atmosphere threshold density is $\rho_{t h r}=1.389 \cdot 10^{-12}$. We label material as unbound in case the fluids 0 th component of the 4 -velocity is smaller than one $u_{t}<-1$ and if the three velocity is radially outward pointing. 
times, can be explained by unbound material reaching the boundary of level 1 , which is the outermost refinement level where matter is evolved. We also note that we see in the bottom panel right panel of Fig. 10 a clear imprint of the refinement boundaries on the low density material. We expect that, as in the $\mathrm{TOV}_{\mathrm{mig}}$ case, the use of the conservative refluxing algorithm would resolve this issue, but postpone this test due to the high computational costs for the presented BNS simulations.

\section{CONCLUSION}

In this article, we have introduced and studied a new method to improve the vacuum treatment for GRHD simulations. Our recipe allows to not set an explicit atmosphere value on the outside of a star which improves the quality of our simulations. Previously, we have implemented a method in the BAM code that used an artificial atmosphere while recovering primitive variables. We extensively tested both methods (vacuum and atmosphere) in single star spacetimes focusing on their performance when combined with a second order local LaxFriedrich and higher-order numerical flux schemes. The use of vacuum methods shows improvement in the mass conservation throughout our simulations. Typically, for the star that forms a low-density region during evolution, the mass conservation drops in the atmosphere method. Up to $0.5 \%$ mass loss was detected in the atmosphere method when the low-density layer crosses the refinement boundary. The violation of mass conservation at the grid refinement boundary does not occur in the case of our improved vacuum method. In most cases, the vacuum method leads to second-order convergence of the mass, in contrast to the atmosphere case, where often second- order convergence is only obtained for a short period of time. Our finding suggests that the use of the vacuum method is desirable and recommended for the single star simulations.

To further investigate the performance of the new vacuum method we presented time evolutions of irrotational equal mass binary neutron star configuration. Mainly, the merger and the postmerger dynamics are of great interest because the artificial atmosphere setup hinders the accurate computation of the ejecta [35. Our analysis suggests that the ejecta materials are better conserved with the vacuum method. Around the moment of merger, the ejected mass, the ejecta velocity, and the kinetic energy of the ejecta are within the same range for both methods (vacuum and atmosphere) for all resolutions. But the difference in those quantities becomes prominent as the ejecta expands to larger radii, and the density of the ejecta drops below the atmosphere threshold. In the atmosphere cases this leads to ejecta removal and does not allow a free expansion of the ejecta material. In contrast, ejected matter can expand freely for the vacuum method.

\section{Acknowledgments}

It is a pleasure to thank S. Bernuzzi and S. V. Chaurasia for fruitful discussions during this project. W.T. was supported by the National Science Foundation under grants PHY-1305387 and PHY-1707227. B.B. was supported by DFG grant BR-2176/5-1. Computations were performed on SuperMUC at the LRZ (Munich) under the project number pr46pu and pn56zo, Jureca (Jülich) under the project number HPO21, and Stampede (Texas, XSEDE allocation - TG-PHY140019).
[1] E. Annala, T. Gorda, A. Kurkela, and A. Vuorinen, Phys. Rev. Lett. 120, 172703 (2018), 1711.02644.

[2] B. P. Abbott et al. (Virgo, LIGO Scientific), Phys. Rev. Lett. 121, 161101 (2018), 1805.11581.

[3] S. De, D. Finstad, J. M. Lattimer, D. A. Brown, E. Berger, and C. M. Biwer, Phys. Rev. Lett. 121, 091102 (2018), 1804.08583.

[4] E. R. Most, L. R. Weih, L. Rezzolla, and J. SchaffnerBielich, Phys. Rev. Lett. 120, 261103 (2018), 1803.00549.

[5] M. W. Coughlin, T. Dietrich, B. Margalit, and B. D. Metzger, Monthly Notices of the Royal Astronomical Society: Letters 489, L91 (2019), ISSN 1745-3925, 1812.04803.

[6] C. D. Capano, I. Tews, S. M. Brown, B. Margalit, S. De, S. Kumar, D. A. Brown, B. Krishnan, and S. Reddy (2019), 1908.10352.

[7] D. Radice and L. Dai, Eur. Phys. J. A55, 50 (2019), 1810.12917.

[8] R. Essick, I. Tews, P. Landry, S. Reddy, and D. E. Holz (2020), 2004.07744.

[9] T. Dietrich, M. W. Coughlin, P. T. Pang, M. Bulla, J. Heinzel, L. Issa, I. Tews, and S. Antier (2020),
2002.11355 .

[10] B. F. Schutz, Nature 323, 310 (1986).

[11] B. P. Abbott et al. (LIGO Scientific, VINROUGE, Las Cumbres Observatory, DLT40, Virgo, 1M2H, MASTER), Nature, 10.1038/nature24471 (2017), 1710.05835.

[12] M. W. Coughlin, T. Dietrich, J. Heinzel, N. Khetan, S. Antier, M. Bulla, N. Christensen, D. A. Coulter, and R. J. Foley, Phys. Rev. Res. 2, 022006 (2020), 1908.00889.

[13] S. Dhawan, M. Bulla, A. Goobar, A. S. Carracedo, and C. N. Setzer (2019), 1909.13810.

[14] M. W. Coughlin, S. Antier, T. Dietrich, R. J. Foley, J. Heinzel, M. Bulla, N. Christensen, D. A. Coulter, L. Issa, and N. Khetan, Nature Commun. 11, 4129 (2020), 2008.07420.

[15] P. S. Cowperthwaite et al., Astrophys. J. 848, L17 (2017), 1710.05840.

[16] S. J. Smartt et al., Nature, 10.1038/nature24303 (2017), 1710.05841 .

[17] M. M. Kasliwal et al., Science 358, 1559 (2017), 1710.05436.

[18] D. Kasen, B. Metzger, J. Barnes, E. Quataert, and 

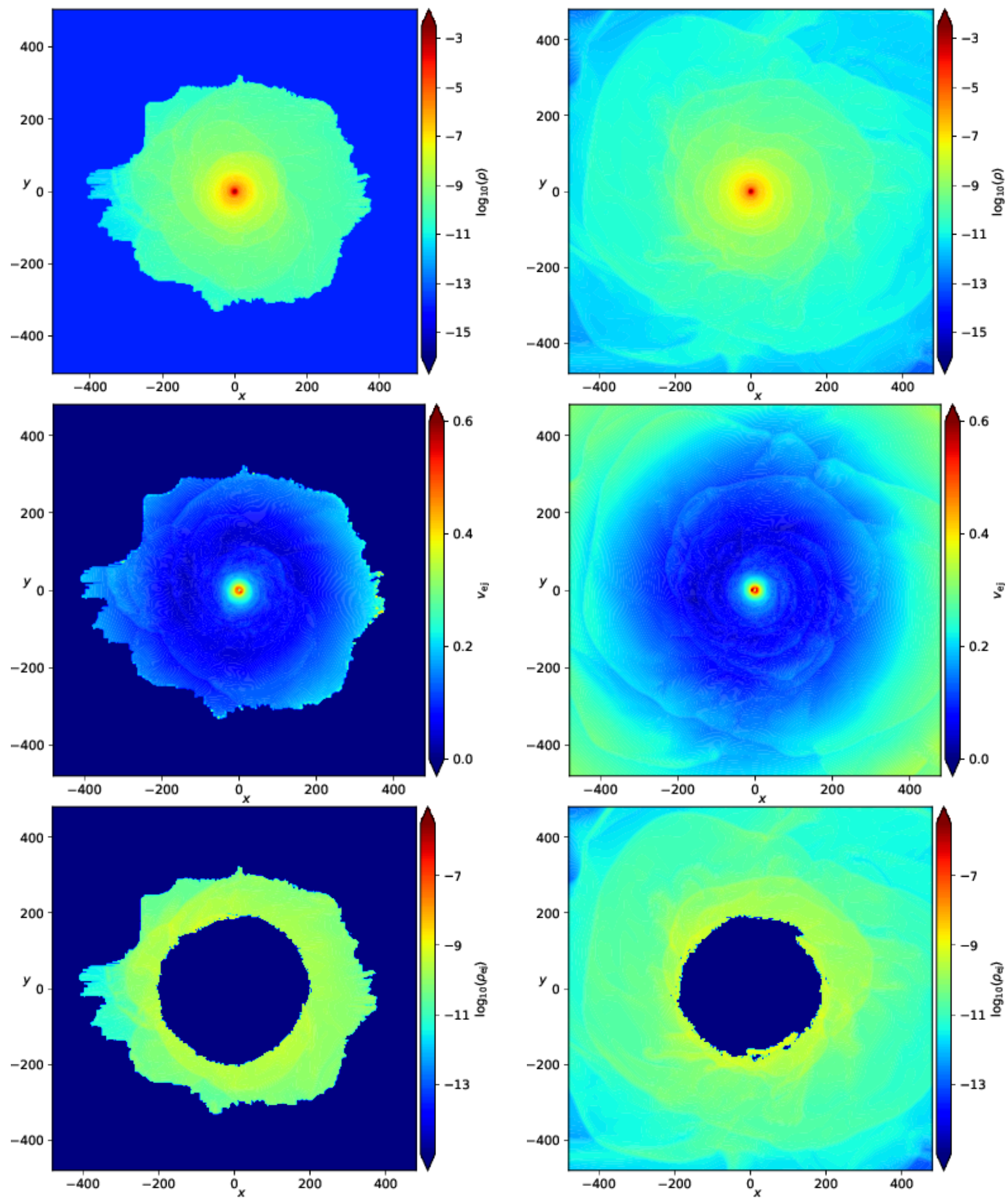

FIG. 11: Snapshots of the mass density (top), velocity (middle), and ejecta mass density (bottom) in xy-plane of BNS simulation. The finest resolution for both the atmosphere (left) and the vacuum (right) at $11 \mathrm{~ms}$ after the merger (vertical cyan line in Fig. 9) is plotted with a linear (velocity) and logarithmic (mass density and ejecta mass density) color scales. 
E. Ramirez-Ruiz, Nature, 10.1038/nature24453 (2017), 1710.05463 .

[19] D. Watson et al., Nature 574, 497 (2019), 1910.10510.

[20] B. P. Abbott et al. (LIGO Scientific, Virgo, Fermi GBM, INTEGRAL, IceCube, AstroSat Cadmium Zinc Telluride Imager Team, IPN, Insight-Hxmt, ANTARES, Swift, AGILE Team, 1M2H Team, Dark Energy Camera GW-EM, DES, DLT40, GRAWITA, Fermi-LAT, ATCA, ASKAP, Las Cumbres Observatory Group, OzGrav, DWF (Deeper Wider Faster Program), AST3, CAASTRO, VINROUGE, MASTER, J-GEM, GROWTH, JAGWAR, CaltechNRAO, TTU-NRAO, NuSTAR, PanSTARRS, MAXI Team, TZAC Consortium, KU, Nordic Optical Telescope, ePESSTO, GROND, Texas Tech University, SALT Group, TOROS, BOOTES, MWA, CALET, IKI-GW Follow-up, H.E.S.S., LOFAR, LWA, HAWC, Pierre Auger, ALMA, Euro VLBI Team, Pi of Sky, Chandra Team at McGill University, DFN, ATLAS Telescopes, High Time Resolution Universe Survey, RIMAS, RATIR, SKA South Africa/MeerKAT), Astrophys. J. 848, L12 (2017), 1710.05833.

[21] J. M. Ezquiaga and M. Zumalacárregui, Phys. Rev. Lett. 119, 251304 (2017), 1710.05901.

[22] T. Baker, E. Bellini, P. G. Ferreira, M. Lagos, J. Noller, and I. Sawicki, Phys. Rev. Lett. 119, 251301 (2017), 1710.06394

[23] P. Creminelli and F. Vernizzi, Phys. Rev. Lett. 119, 251302 (2017), 1710.05877.

[24] M. Alcubierre, Introduction to 3+1 Numerical Relativity (Oxford University Press, New York, 2008), ISBN 978-019-920567-7.

[25] T. W. Baumgarte and S. L. Shapiro, Numerical Relativity, Solving Eisntein's Equations on the Computer (Cambridge University Press, New York, 2010), ISBN 978-0521-51407-1.

[26] L. Rezzolla and O. Zanotti, Relativistic Hydrodynamics (Oxford University Press, 2013).

[27] B. P. Abbott et al. (LIGO Scientific, Virgo), Phys. Rev. Lett. 119, 161101 (2017), 1710.05832.

[28] B. P. Abbott et al. (LIGO Scientific, Virgo, FermiGBM, INTEGRAL), Astrophys. J. 848, L13 (2017), 1710.05834 .

[29] T. Yamamoto, M. Shibata, and K. Taniguchi, Phys.Rev. D78, 064054 (2008), 0806.4007.

[30] M. Thierfelder, S. Bernuzzi, and B. Brügmann, Phys. Rev. D84, 044012 (2011), 1104.4751.

[31] L. Baiotti and L. Rezzolla, Rept. Prog. Phys. 80, 096901 (2017), 1607.03540.

[32] D. Radice, L. Rezzolla, and F. Galeazzi, Class.Quant.Grav. 31, 075012 (2014), 1312.5004.

[33] J. R. Westernacher-Schneider, C. Markakis, and B. J. Tsao, Class. Quant. Grav. 37, 155005 (2020), 1912.03701.

[34] B. Brügmann, J. González, M. Hannam, S. Husa, U. Sperhake, and W. Tichy, Phys. Rev. D77, 024027 (2008), gr-qc/0610128.

[35] T. Dietrich, S. Bernuzzi, M. Ujevic, and B. Brügmann, Phys. Rev. D91, 124041 (2015), 1504.01266.

[36] S. Bernuzzi and T. Dietrich, Phys. Rev. D94, 064062 (2016), 1604.07999.

[37] T. Dietrich, S. Ossokine, and K. Clough, Class. Quant. Grav. 36, 025002 (2019), 1807.06959.

[38] Z.-j. Cao, H.-J. Yo, and J.-P. Yu, Phys. Rev. D78, 124011 (2008), 0812.0641.

[39] B. Brügmann, W. Tichy, and N. Jansen, Phys. Rev. Lett.
92, 211101 (2004), gr-qc/0312112.

[40] D. Pollney, C. Reisswig, E. Schnetter, N. Dorband, and P. Diener (2009), 0910.3803.

[41] J. Thornburg, in The Ninth Marcel Grossman Meeting: On Recent Developments in Theoretical and Experimental General Relavtivity, Gravitation, and Relativistic Field Theories, edited by V. G. Gurzadyan, R. T. Jantzen, and R. Ruffini (World Scientific, Singapore, 2003), pp. 1743-1744, gr-qc/0012012.

[42] J. Thornburg, Class. Quantum Grav. 21, 3665 (2004), grqc/0404059, URL http://stacks.iop.org/0264-9381/ $21 / 3665$

[43] M. Ruiz, D. Hilditch, and S. Bernuzzi, Phys. Rev. D83, 024025 (2011), 1010.0523.

[44] D. Hilditch, S. Bernuzzi, M. Thierfelder, Z. Cao, W. Tichy, et al., Phys.Rev. D88, 084057 (2013), 1212.2901

[45] S. Bernuzzi and D. Hilditch, Phys. Rev. D81, 084003 (2010), 0912.2920.

[46] C. Bona, J. Massó, E. Seidel, and J. Stela, Phys. Rev. Lett. 75, 600 (1995), gr-qc/9412071.

[47] M. Alcubierre, B. Brügmann, P. Diener, M. Koppitz, D. Pollney, E. Seidel, and R. Takahashi, Phys. Rev. D 67, 084023 (2003), gr-qc/0206072.

[48] J. R. van Meter, J. G. Baker, M. Koppitz, and D.-I. Choi (2006), gr-qc/0605030, gr-qc/0605030.

[49] A. Sommerfeld, Partial differential equation in physics (Academic Press, New York, 1949).

[50] F. Banyuls, J. A. Font, J. M. Ibánez, J. M. Mart 'i, and J. A. Miralles, Astrophys. J. 476, 221 (1997).

[51] E. Chabanat, J. Meyer, P. Bonche, R. Schaeffer, and P. Haensel, Nucl. Phys. A627, 710 (1997).

[52] F. Douchin and P. Haensel, Astron. Astrophys. 380, 151 (2001), astro-ph/0111092.

[53] M. Shibata, K. Taniguchi, and K. Uryu, Phys.Rev. D71, 084021 (2005), gr-qc/0503119.

[54] A. Bauswein, H.-T. Janka, and R. Oechslin, Phys.Rev. D82, 084043 (2010), 1006.3315.

[55] J. A. Font, M. Miller, W. M. Suen, and M. Tobias, Phys. Rev. D 61, 044011 (2000), gr-qc/9811015.

[56] H. Dimmelmeier, J. A. Font, and E. Müller, Astron. Astrophys. 388, 917 (2002).

[57] L. Baiotti, I. Hawke, P. J. Montero, F. Löffler, L. Rezzolla, N. Stergioulas, J. A. Font, and E. Seidel, Phys. Rev. D 71, 024035 (2005), gr-qc/0403029.

[58] R. Borges, M. Carmona, B. Costa, and W. S. Don, Journal of Computational Physics 227, 3191 (2008), ISSN 0021-9991, URL http://www.sciencedirect.com/ science/article/pii/S0021999107005232

[59] C. Bona, J. Massó, and J. Stela, Phys. Rev. D 51, 1639 (1995).

[60] M. Hannam, S. Husa, F. Ohme, B. Brügmann, and N. O'Murchadha, Phys. Rev. D78, 064020 (2008), 0804.0628.

[61] T. Dietrich and B. Brügmann, J. Phys. Conf. Ser. 490, 012155 (2014), 1403.5746.

[62] M. Thierfelder, S. Bernuzzi, D. Hilditch, B. Brügmann, and L. Rezzolla, Phys.Rev. D83, 064022 (2011), 1012.3703 .

[63] A. Kurganov and E. Tadmor, J. Comp. Phys. 160, 214 (2000).

[64] A. Lucas-Serrano, J. A. Font, J. M. Ibanez, and J. M. Marti, Astron. Astrophys. 428, 703 (2004), astro$\mathrm{ph} / 0407541$. 
[65] H. Nessyahu and E. Tadmor, J. Comp. Phys. 87, 408-463 (1990).

[66] J. A. Font, T. Goodale, S. Iyer, M. Miller, L. Rezzolla, E. Seidel, N. Stergioulas, W.-M. Suen, and M. Tobias, Phys. Rev. D 65, 084024 (2002).

[67] M. J. Berger and P. Colella, J. Comp. Phys. 82, 64 (1989).

[68] B. Giacomazzo and R. Perna, Astrophys. J. 758, L8 (2012), 1209.0783.

[69] C. Reisswig, R. Haas, C. D. Ott, E. Abdikamalov, P. Mösta, D. Pollney, and E. Schnetter, Phys. Rev. D 87, 064023 (2013), 1212.1191.

[70] T. Dietrich and S. Bernuzzi, Phys. Rev. D91, 044039 (2015), 1412.5499.

[71] J. S. Read, B. D. Lackey, B. J. Owen, and J. L. Friedman, Phys.Rev. D79, 124032 (2009), 0812.2163.

[72] W. Tichy, Phys. Rev. D74, 084005 (2006), grqc/0609087.
[73] W. Tichy, Class. Quant. Grav. 26, 175018 (2009), 0908.0620

[74] T. Dietrich, N. Moldenhauer, N. K. Johnson-McDaniel, S. Bernuzzi, C. M. Markakis, B. Brügmann, and W. Tichy, Phys. Rev. D92, 124007 (2015), 1507.07100.

[75] A. Savitzky and M. J. E. Golay, Analytical Chemistry 36, 1627 (1964)

[76] A. Savitzky, Analytical Chemistry 61, 921A (1989), pMID: 22882064, https://doi.org/10.1021/ac00190a744, URL https://doi.org/10.1021/ac00190a744

[77] N. Stergioulas, A. Bauswein, K. Zagkouris, and H.-T. Janka, Mon. Not. Roy. Astron. Soc. 418, 427 (2011), 1105.0368 .

[78] L. Rezzolla and K. Takami, Phys. Rev. D 93, 124051 (2016), 1604.00246.

[79] B. D. Metzger, Living Rev. Rel. 20, 3 (2017), 1610.09381. 